BRE 23584

\title{
Feline subthalamic nucleus neurons contain glutamate-like but not GABA-like or glycine-like immunoreactivity
}

\author{
Roger L. Albin, J. Wayne Aldridge, Anne B. Young and Sid Gilman \\ Department of Neurology, University of Michigan, Ann Arbor, MI 48104 (U.S.A.)
}

(Accepted 13 March 1989)

Key words: Subthalamic nucleus; Glutamate; $\gamma$-Aminobutyric acid; Glycine; Immunohistochemistry

\begin{abstract}
The identity of the neurotransmitter of subthalamic nucleus neurons has not been definitively established. GABA, glycine, and glutamate have all been hypothesized to be the neurotransmitter of these neurons. Immunohistochemistry with 3 well characterized antisera against glutamate, GABA, and glycine were used to study feline subthalamic nucleus neurons. These neurons were found to contain intense glutamate-like but not GABA- or glycine-like immunoreactivity. The surrounding neuropil contained glutamate-like and GABA-like but not glycine-like immunoreactivity. These results support the hypothesis that subthalamic nucleus neurons are glutamatergic.
\end{abstract}

The subthalamic nucleus (STN) plays an important role in the control of motor behavior. Lesions of the STN in both human and non-human primates and pharmacological inhibition of its activity in non-human primates give rise to a profoundly disabling hyperkinetic movement disorder termed hemiballism ${ }^{2}$. Virtually all STN neurons are projection neurons ${ }^{30}$. STN neurons are uniquely positioned to modulate the output of the basal ganglia because they receive afferents from lateral globus pallidus, sensorimotor cortex, and centre-median/parafascicular complex and project to entopeduncular nucleus (or its primate homologue, medial globus pallidus) and substantia nigra pars reticulata $(\mathrm{SNr})^{17}$, 27. While the importance of the STN in motor function has been recognized for decades ${ }^{10}$, the identity of the neurotransmitter of STN neurons has not been defined. Physiological studies with extracellular recording techniques showed that STN stimulation produced excitation of neurons in the $\mathrm{SNr}^{6,7}$, and inhibition of neurons in the entopeduncular nucleus and globus pallidus ${ }^{12,18,24,32}$. These results appear to be paradoxical as physiological and anatomical studies have established that STN projection neurons send branched axons to both the pallidum and the substantia nigra ${ }^{3,30}$. The observation of inhibition of pallidal neurons by STN stimulation led to suggestions that $\gamma$-aminobutyric acid (GABA) ${ }^{24}$ or glycine $(\mathrm{Gly})^{31}$ is the neurotransmitter of STN neurons. This inhibitory response could be abolished by the GABA antagonists picrotoxin and bicuculline ${ }^{24}$, indicating that GABA might be responsible for the inhibition of pallidal neurons. In addition, the finding that $\left[{ }^{3} \mathrm{H}\right] \mathrm{GABA}$ was transported into STN neurons when injected into the pallidal complex of cats ${ }^{15}$ and the globus pallidus ${ }^{28}$ of rats suggested that GABA was indeed the neurotransmitter of STN neurons.

The conclusion that GABA is the neurotransmitter of STN neurons has been undermined by a series of recent studies. Immunohistochemical experiments with antisera directed against glutamic acid decarboxylase (GAD), the synthetic enzyme for GABA, and GABA itself, have failed to reveal evidence of $\mathrm{GABA}^{25,26}$ - or $\mathrm{GAD}^{13}$-immunoreactive STN neurons. An in situ hybridization study with a ribonucleotide probe for GAD failed to demonstrate that STN neurons contain GAD mRNA ${ }^{1}$. A recent study of STN efferent projections in rat showed that subthalamopallidal terminals contain round vesicles

Correspondence: R.L. Albin, Neuroscience Laboratory Building, 1103 E. Huron, Ann Arbor, MI 48104, U.S.A. 
and form asymmetrical synapses, whereas previous studies had shown that GABAergic boutons within the pallidum contain pleomorphic vesicles and form symmetric synapses ${ }^{11}$. No previous study has used immunocytochemical techniques to test the hypothesis that Gly is the neurotransmitter of the STN.

Nakanishi et al. have recently used intracellular recording techniques to demonstrate that STN stimulation gives rise to monosynaptic excitatory postsynaptic potentials in $\mathrm{SNr}$ neurons, suggesting that the neurotransmitter of STN is excitatory ${ }^{14}$. Extracellular recordings in the medial globus pallidus (MGP) of non-human primates after STN lesions have revealed a decrease in unit activity, supporting the hypothesis that the STN exerts an excitatory influence on MGP neurons ${ }^{5}$. A hyperkinetic movement disorder identical to that produced by inhibition of STN function in rhesus monkeys ${ }^{2}$ has been induced in rhesus monkeys by injection of the excitatory amino acid antagonist kynurenic acid into the MGP ${ }^{21}$. This latter observation suggests that the neurotransmitter of STN neurons may be an excitatory amino acid. The hypothesis that an excitatory amino acid is the neurotransmitter of STN neurons is buttressed by reports that STN neurons in rodents and squirrel monkeys contain glutamate-like immunoreactivity ${ }^{16.25}$.

To explore further the identity of the STN neuron neurotransmitter we undertook an immunohistochemical study of feline STN neurons using antisera directed against the putative excitatory amino acid neurotransmitter glutamate (Glu) and the inhibitory amino acid neurotransmitters GABA and Gly.

Two adult cats were deeply anesthetized with pentobarbital and perfused transcardially with 1.0 liter of $0.9 \% \mathrm{NaCl}$ solution followed by 3.5 liters of $4 \%$ paraformaldehyde $-0.2 \%$ glutaraldehyde- $0.1 \mathrm{M}$ sodium phosphate buffer ( $\mathrm{pH}$ 7.4). After extraction from the cranial vault, the brains were postfixed overnight in the same fixative at $4{ }^{\circ} \mathrm{C}$. The brains were then serially immersed in 10,20 and 30\% sucrose in $0.1 \mathrm{M}$ phosphate buffer. One of these animals had received an ibotenic acid lesion of the right medial geniculate nucleus while the other had received a lesion of the right STN. These lesions were placed as part of a study on the effects of STN ablation on single unit activity in the pallidum and did not affect the present observations. Forty $-\mu \mathrm{m}$ thick frozen sections were cut on a sliding microtome and stored in $0.1 \mathrm{M}$ phosphate buffer plus $0.01 \%$ NaAzide until the time of assay. Three well charac-

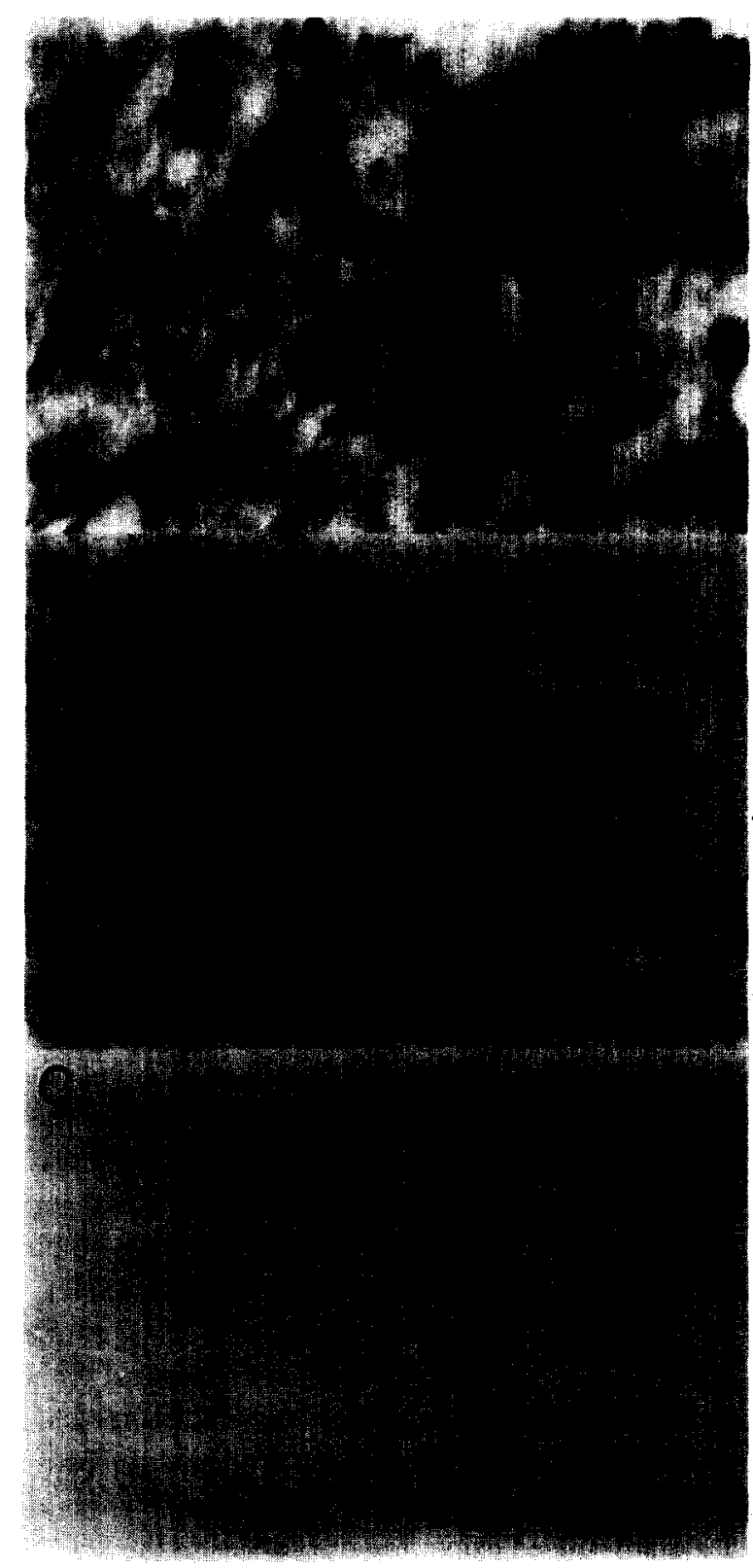

Fig. 1. Feline subthalamic nucleus neurons stained with anti-glutamate (A), anti-GABA (B), and anti-glycine (C) antisera. With the anti-glutamate antisera, neurons are robustly stained and the surrounding neuropil is stained as well. The anti-GABA antiserum produces weaker cell staining that outlines some STN neuronal perikarya (arrow in B). Neuropil staining is also evident with the anti-GABA staining. No glycine-like immunoreactivity is present in either neurons or neuropil. Bar $=80 \mu \mathrm{m}$. 
terized polyclonal antisera directed against glutamate ${ }^{9}, \mathrm{GABA}^{8}$, and $\mathrm{Gly}^{19}$ were utilized. The concentrations of primary antisera were $1 / 1000$ for the glutamate antiserum, $1 / 1000$ for the GABA antiserum, and $10.4 \mu \mathrm{g} / \mathrm{ml}$ for the Gly antiserum. Free floating sections were processed with the peroxidase-antiperoxidase technique as previously described $^{20}$.

Examination of sections exposed to the Glu antiserum revealed an abundance of intensely immunoreactive STN neurons (Fig. 1A). While quantitation was not performed, it appeared that virtually all STN neurons contained Glu-like immunoreactivity. The Glu-like immunoreactivity appeared to fill the cytoplasm and proximal neuronal processes. In sections exposed to the GABA or Gly antiserum no STN neurons contained GABA-like or Gly-like immunoreactivity (Fig. 1B and C). As previously reported by Smith and Parent in monkeys ${ }^{25}$, the STN neuropil was also positively stained by the Glu and GABA antisera. The STN contained large numbers of Glu-like immunoreactive fibers and GABA-like immunoreactive puncta that seemed to surround STN neurons. No Gly-like immunoreactivity of any kind was present in the STN. Methodological control sections were run by omitting the primary antisera or substituting non-immune rabbit serum for primary antiserum. No staining was seen under these control conditions. The Gly antiserum positively stained neurons and neuropil in sections of cervical spinal cord. The GABA antiserum stained neurons and

1 Chesselet, M.-F., Weiss, L., Wuenschell, C., Tobin, A.J. and Affolter, H.-U., Comparative distribution of mRNAs for glutamic acid decarboxylase, tyrosine hydroxylase, and tachykinins in the basal ganglia: an in situ hybridization study in the rodent brain, J. Comp. Neurol., 262 (1987) 125-140.

2 Crossman, A.R., Primate models of dyskinesia: the experimental approach to the study of basal ganglia-related involuntary movement disorders, Neuroscience, 21 (1987) $1-40$.

3 Deniau, J.M., Hammond, C., Chevalier, G. and Feger, J., Evidence for branched subthalamic nucleus projections to substantia nigra, entopeduncular nucleus, and globus pallidus, Neurosci. Lett., 9 (1978) 117-121.

4 Fonnum, F., Grofova, I. and Rinvik, E., Origin and distribution of glutamate decarboxylase in the nucleus subthalamicus of the cat, Brain Research, 153 (1978) 370-374.

5 Hamada, I. and DeLong, M.R., Lesions of the primate subthalamic nucleus reduce tonic and phasic neural activity in globus pallidus, Soc. Neurosci. Abstr., 14 (1988) 719. neuropil in the molecular layer of the cerebellum.

Our finding of Glu-like and GABA-like immunoreactive STN neuropil is consistent with evidence that afferents to the STN use these substances as neurotransmitters. While the Glu-like immunoreactive fibers could be recurrent collaterals, recent physiological data suggest that the neurotransmitter of the cortico-STN projection is an excitatory amino $\operatorname{acid}^{23}$. The presence of GABA-like immunoreactive puncta is consistent with biochemical and neurochemical evidence that GABA is the neurotransmitter of the pallidal-STN projection ${ }^{4,22}$. Recently, Takada and Hattori described retrograde transport of $\left[{ }^{3} \mathrm{H}\right]-$ Gly by rat pallido-STN projection neurons and suggested that Gly is the neurotransmitter of the pallidal-STN projection ${ }^{28}$. Their conclusion is not supported by our results and a previous study in rats that demonstrated a paucity of Gly-like immunoreactive neurons and fibers in the forebrain ${ }^{29}$.

As with previous immunohistochemical studies in rodents and non-human primates, our findings support the hypothesis that the neurotransmitter of STN neurons is an excitatory amino acid.

We would like to thank Drs. Aldo Rustioni and Roberta Pourcho for the gifts of the anti-glutamate and anti-glycine antisera, respectively. We are indebted to Kevin Kaatz and Jill Johnson for efficient technical assistance. This work was supported by NIH Grants NS 19613 and KO8 NS01300.

6 Hammond, C., Deniau, J.M., Rizk, A. and Feger, J., Electrophysiological demonstration of an excitatory subthalamonigral pathway in the rat, Brain Research, 151 (1978) 235-244.

7 Hammond, C., Shibazaki, T. and Rouzaire-Dubois, B., Branched output neurons of the rat subthalamic nucleus: electrophysiological study of the synaptic effects on identified cells in the two main target nuclei, the entopeduncular nucleus and the substantia nigra, Neuroscience, 9 (1983) 511-520.

8 Hendry, S.H.C., Schwark, H.D., Jones, E.G. and Yan, J., Numbers and proportions of GABA-immunoreactive neurons in different areas of monkey cerebral cortex, $J$. Neurosci., 7 (1987) 1503-1519.

9 Hepler, J.R., Toomim, C.S., McCarthy, K.D., Conti, F., Battaglia, G., Rustioni, A. and Petrusz, P., Characterization of antisera to glutamate and aspartate, J. Histochem. Cytochem., 36 (1988) 13-22.

10 Jakob, A., Arteriosklerotische Muskelstarre mit hinzutretendem Hemiballismus. In O. Foerster and K. Wilmanns (Eds.), Die Extrapyramidalen Erkrankungen, Elsevier, 
New York, 1923, pp. 183-225.

11 Kita, H. and Kitai, S.T., Efferent projections of the subthalamic nucleus in the rat: light and electron microscopic analysis with the PHA-L method, J. Comp. Neurol., 260 (1987) 435-452.

12 Larsen, K.D. and Sutin, J., Output organization of the feline entopeduncular and subthalamic nuclei, Brain Research, 157 (1978) 21-31.

13 Mugnaini, E. and Oertel, W.H., An atlas of the distribution of GABAergic neurons and terminals in the rat CNS as revealed by GAD immunocytochemistry in the rat. In A. Björklund and T. Hökfelt (Eds.), Handbook of Chemical Neuroanatomy, GABA and Neuropeptides in the CNS, Vol. 4, Part I, Elsevier, Amsterdam, 1985, pp. 436-508.

14 Nakanishi, H., Kita, H. and Kitai, S.T., Intracellular study of rat substantia nigra pars reticulata neurons in an in vitro slice preparation: electrical membrane properties and response characteristics to subthalamic stimulation, Brain Research, 437 (1987) 45-55.

15 Nauta, H.J.W. and Cuenod, M., Perikaryal cell labelling in the subthalamic nucleus following the injection of $\left[{ }^{3} \mathrm{H}\right]$ GABA in the pallidal complex: an autoradiographic study in the cat, Neuroscience, 7 (1982) 2725-2734.

16 Ottersen, O.P. and Storm-Mathisen, J., Glutamate- and GABA-containing neurons in the mouse and rat brain as demonstrated with a new immunocytochemical technique, J. Comp. Neurol, 229 (1984) 374-392.

17 Parent, A., Comparative Neurobiology of the Basal Ganglia, Wiley, New York, 1986.

18 Perkins, M.N. and Stone, T.W., Subthalamic projections to the globus pallidus: an electrophysiological study in the rat, Exp. Neurol., 68 (1980) 500-511.

19 Pourcho, R.G. and Goebel, D.J., Immunocytochemical demonstration of glycine in retina, Brain Research, 348 (1985) 339-342.

20 Reiner, A., Brecha, N. and Karten, H., Enkephalinmediated basal ganglia influences over the optic tectum: immunohistochemistry of the tectum and the lateral spiriform nucleus in pigeons, J. Comp. Neurol., 208 (1982) $37-53$.

21 Robertson, R.G., Farmery, S.M., Sambrook, M.A. and Crossman, A.R., Dyskinesia in the primate following injection of an excitatory amino acid antagonist into the medial segment of the globus pallidus, Brain Research, 476 (1989) 317-322

22 Rouzaire-Dubois, B., Hammond, C., Hamon, B. and Feger, J., Pharmacological blockade of the globus pallidusinduced inhibitory response of subthalamic cells in the rat, Brain Research, 200 (1980) 321-329.

23 Rouzaire-Dubois, B. and Scarnati, E., Pharmacological study of the cortical-induced excitation of subthalamic nucleus neurons in the rat: evidence for amino acids as putative neurotransmitters, Neuroscience, 21 (1987) 429440.

24 Rouzaire-Dubois, B., Scarnati, E., Hammond, C., Crossman, A.R. and Shibazaki, T., Microiontophoretic studies on the nature of the neurotransmitter in the subthalamicentopenduncular pathway of the rat, Brain Research, 271 (1983) $11-20$

25 Smith, Y. and Parent, A., Neurons of the subthalamic nucleus in primates display glutamate but not GABA immunoreactivity, Brain Reseaich, 453 (1988) 353-356.

26 Smith, Y., Parent, A., Seguela, P. and Descarries, L., Distribution of GABA-immunoreactive neurons in the basal ganglia of the squirrel monkey (Samiri sciureus), $J$. Comp. Neurol., 259 (1988) 50-64.

27 Sugimoto, T., Hattori, H., Mizuno, N., Itoh, K. and Sabo, M., Direct projections from the centre median-parafascicular complex to the subthalamic nucleus in the cat and rat, J. Comp. Neurol., 214 (1983) 209-214.

28 Takada. M. and Hattori, T., Glycine: an alternative transmitter candidate of the pallidosubthalamic projection neurons in the rat, J. Comp. Neurol., 262 (1987) 465-472.

29 Van den Pol, A. and Gorcs, T., Glycine and glycine receptor immunoreactivity in brain and spinal cord, $J$. Neurosci., 8 (1988) 472-492.

30 Van der Kooy, D. and Hattori, T., Single subthalamic nucleus neurons project to both the globus pallidus and substantia nigra in rat, J. Comp. Neurol., 192 (1980) 751-768.

31 Yoshida, M., Functional aspect of, and role of transmitter in, the basal ganglia, Confin. Neurol., 36 (1974) 282-291.

32 Yoshida, M., Rabin, A. and Anderson, M., Two types of monosynaptic inhibition of pallidal neurons produced by stimulation of the diencephalon and substantia nigra, Brain Research, 30 (1971) 235-239. 